\title{
Estimating Trust Value for Cloud Service Providers using Fuzzy Logic
}

\author{
Supriya M, Venkataramana L.J, K Sangeeta \\ Department of Computer Science and Engineering, \\ Amrita School of Engineering \\ Kasavanahalli, Bengaluru -560035
}

\author{
G K Patra \\ Centre for Mathematical Modelling and Computer \\ Simulations, Council of Scientific and Industrial \\ Research \\ Bengaluru-560037
}

\begin{abstract}
Cloud computing has garnered popular support in a relatively short span of time. It is a new method of delivering the distributed resources over internet. It reduces capital expenditure as well as operational expenditure. The number of cloud service providers (CSPs) who provide computing as a utility has increased exponentially in the past few years, providing more options for the customers to choose from. In this paper a model for Trust Management based on Fuzzy Logic has been developed, which can help consumers make an informed choice towards selecting the appropriate CSP as per their requirement.
\end{abstract}

\section{General Terms}

Cloud Model, Trust Management

\section{Keywords}

Cloud Service Provider, Trust, Fuzzy Logic, Cloud Analyst.

\section{INTRODUCTION}

Distributed computing was developed with the aim to dispose of the need to have expensive supercomputers to solve the problems faced by scientific community. Cloud computing is the latest form of evolution of distributed computing. Cloud Computing can be defined as a model for enabling ubiquitous, convenient, on-demand network access to a shared pool of configurable computing resources (e.g., networks, servers, storage, applications, and services) that can be rapidly provisioned and released with minimal management effort or service provider interaction [1]. The services offered by cloud computing are classified as - Infrastructure as a Service (Iaas), Platform as a Service (Paas), Software as a Service (Saas) and the deployment models are classified as private, community, public and hybrid cloud [2].

Cloud computing has evolved into a business idea where cloud service providers (CSPs) provide computing as an utility, which needs to be paid as per the usage. This is analogous to paying for the electricity consumed to the Electricity Board (E.B). The E.B takes care of production, maintenance and transportation for the electricity, the consumer just needs to pay the bill based on one's usage. However cloud is far from being perfect. Like any service it needs proper guidelines to maintain standards and integrity. Since it is a common platform for various parties it becomes increasingly difficult to maintain safety standards. Also, the number of CSPs has gone up in recent years so that the customer has to make a judicious choice based on various parameters such as cost, security, performance etc.

Hassan et al [3] have published a survey on Trust and Trust Management in Cloud Computing. This paper analyzes the trust management systems proposed for cloud computing by various researchers with special emphasis on their capability, their applicability in practical heterogenous cloud environment and their implementabilty. Sun et al [4] have proposed a trust management model based on fuzzy set theory named TMFC. Here the direct and recommended trust measurements using the concept of fuzzy set theory have been discussed. Their proposed model provide a helpful measure to enhance the robustness, fault tolerance and security of cloud computing. However, it doesn't define the trust evaluation attributes of the CSP. Alhamad et al [5] have described a model for scalability, availability, security and usability parameters of trust for Iaas using fuzzy-set theory. This paper uses Sugeno fuzzy-inference approach for developing an overall trust rating for a given CSP.

Cloud Service Measurement Index Consortium (CSMIC) [6] proposes a framework based on common characteristics of cloud services. The aim of this consortium is to define each of QoS attributes given in the framework and provide a methodology for computing a relative index for comparing different cloud services. CSMIC has developed the Service Measurement Index (SMI) which consists of a set of Key Performance Indicators (KPI) that helps to standardize the measurement of business services. SMICloud - A work published by Garg et al [7] has proposed a framework to measure the quality of CSPs and prioritize them, which will create healthy competition among cloud providers to satisfy their Service Level Agreement (SLA) and improve their Quality of Services (QoS). SMICloud, systematically measures all the QoS attributes proposed by CSMIC and rank the cloud services based on these attributes. The threats and security issues in cloud computing are discussed in [8], [9]. Thus, cloud computing has opened up a new frontier of challenges and the problem of trusting cloud computing is of supreme concern for most enterprises. In order to address a few of these issues related to trusting the Cloud Service Providers, in this paper we propose a model which would help the users of cloud to make an informed choice based on their requirements.

The paper is organized as follows: Section 2 describes the proposed trust model and the block diagram to estimate the trust value for CSP based on the identified parameters. Implementation details for the assumed scenario are discussed in section 3 and conclusion in section 4 .

\section{MODEL DESCRIPTION}

Trust is strongly connected to confidence and it implies some degrees of uncertainty, hopefulness or optimism. The most relevant sources of information considered by the trust and reputation models presented before, are direct experiences and witness information. The direct experience is considered as direct trust and the witness information is considered as recommended trust. Based on the location or limit within which 
the trust is estimated, the trust is classified as Inter Domain and

\subsection{The Trust Model}

Our model architecture concentrates on the estimation of trust value for CSP in Inter and Intra Domain and is based on the Direct and Recommended information. Figure 1 shows the overall architecture of the trust estimation.

In this paper, we have simulated an environment using Cloud Analyst and focused on the estimation of Inter Domain trust value for CSP based on the direct relationship.

Cloud Analyst is developed by Bhathiya Wickremasinghe et al. [10] at the CLOUDS Laboratory. It is built on top of CloudSim and separates the simulation experimentation from a programming task enabling one to concentrate on the simulation parameters rather than the technicalities of programming.

Simulation in Cloud Analyst involves the following steps

\section{i. Defining and configuration of User Bases. \\ ii. Defining and configuring Data Centers \\ iii. Allocating of Virtual Machines in Data Centers. \\ iv. Review and Adjustment of various other parameters such as Packet size, No of packets, Bandwidth, and Load balancing policies}

The Cloud Analyst enables us to model different scenarios of CSPs and User Bases, and provides a comprehensive output detailing the response time, Data Center processing time and total cost involved in the communication and computation. Figure 2 shows the snapshot of the Cloud Analyst's configuration window.
Intra Domain.

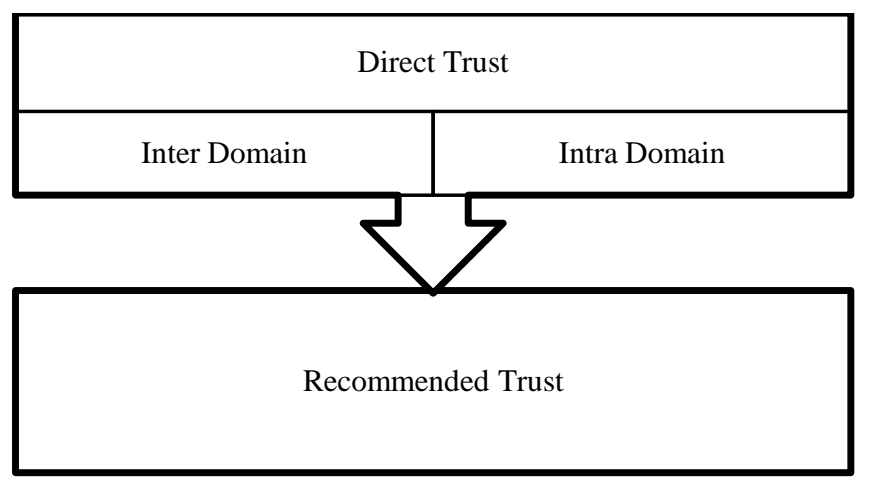

Figure 1: The Overall Architecture

\subsection{Model Parameters}

The model parameters are chosen based on the attributes defined by Service Measurement Index (SMI) [7]. These include Accountability, Agility, Assurance, Financial, Performance, Security, Privacy, and Usability. Each of these attributes consists of a set of Key Performance Indicators (KPIs) which describe the data to be collected for measurement. KPIs are quantifiable measurements, agreed to beforehand, that reflect the critical success factors of an organization. They will differ depending on the organization. However, of the KPIs, not all are measurable i.e. quantifiable, some are qualitative in nature. Based on the KPI that make up the attributes in evaluating the CSPs, we discuss Performance, Financial and Agility, in this paper. Table 1 shows the factors and their KPI.

\section{Configure Simulation}

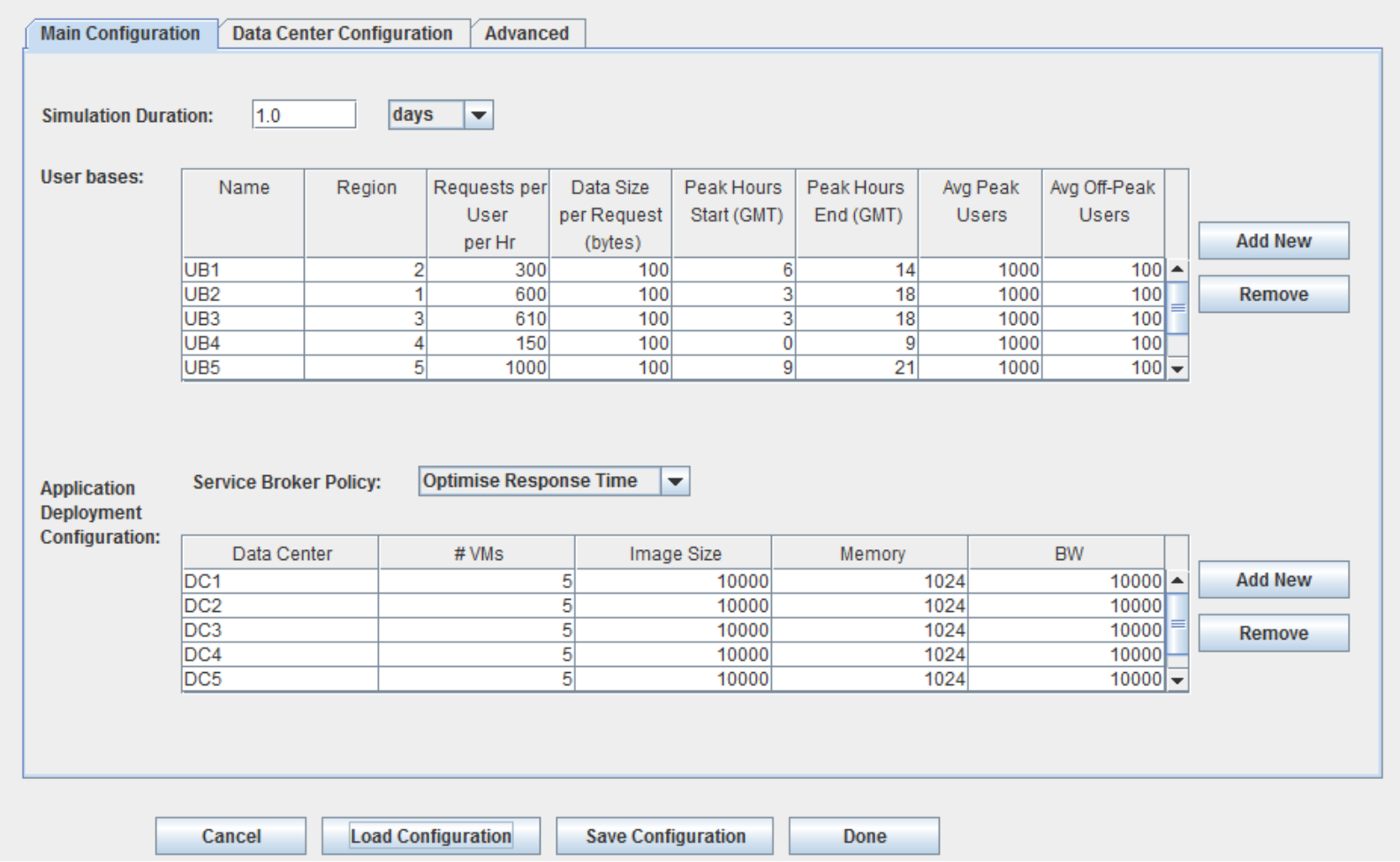

Figure 2: Cloud Analyst configuration window 
Table 1: Factors and their KPI

\begin{tabular}{|l|l|}
\hline $\begin{array}{l}\text { Factors Impacting } \\
\text { Degree of Trust }\end{array}$ & KPIs of the corresponding attribute \\
\hline Performance & $\begin{array}{l}\text { Accuracy, Functionality, Stability, } \\
\text { Interoperability, Service Response } \\
\text { Time }\end{array}$ \\
\hline Financial & $\begin{array}{l}\text { Acquisition and training cost, } \\
\text { Ongoing cost, Profit or Cost } \\
\text { Sharing }\end{array}$ \\
\hline Agility & $\begin{array}{l}\text { Adaptability, Capacity, Elasticity, } \\
\text { Extensibility, Flexibility, } \\
\text { Portability, Scalability }\end{array}$ \\
\hline
\end{tabular}

The evaluation of the trust value for CSP comprises of two stages as shown in Figure 3. The first stage is the implementation with the help of Mamdani Fuzzy Inference System [11]. It takes Performance, Financial and Agility as inputs and produces a range of values which could be easily fed as input to the next level of processing. The Performance attribute is evaluated by passing Data Center (DC) Processing Time, Processor Speed and User Base (UB) Response Time as inputs to the Mamdani FIS. Financial attribute is calculated with the following inputs: Virtual Machine (V.M) Cost, Memory Cost, Storage Cost, and Data Transfer Cost. Finally, Agility attribute has number of Physical Units, Memory Size, and number of V.Ms as its inputs.
The second stage is the implementation using Sugeno FIS. It takes the output of the Mamdani FIS and helps to obtain the trust rating for the CSP.

For both the FIS, the membership values for performance, financial and agility parameters are assumed as low, medium, high and very high as per the requirement. For example, certain input parameters can have values only in a short interval while some may vary over larger range.

The above two stages are implemented hierarchically using the fuzzy logic blocks in Simulink of MATLAB [11]

\section{IMPLEMENTATION AND RESULTS}

The implementation consists of two stages. First is simulating the cloud environment, next is using the parameters from the simulation in Fuzzy Logic toolbox to obtain the trust rating.

\subsection{Simulation setup}

In Cloud Analyst, the scenarios are setup in such a way as to represent user bases across the globe. The user bases remain constant across the scenarios whereas the CSP's setup changes. So for the same amount of user load we are able to determine the performance of various CSPs. We take an example scenario with five different CSPs each with unique setting representing the geographic diversity, the cost factor, and the processing capabilities.

Table 2 describes the values which are taken to simulate the cloud environment using Cloud Analyst. So, five different simulations are run and each produces an output report detailing the Response time, Data Center processing time and the total cost.

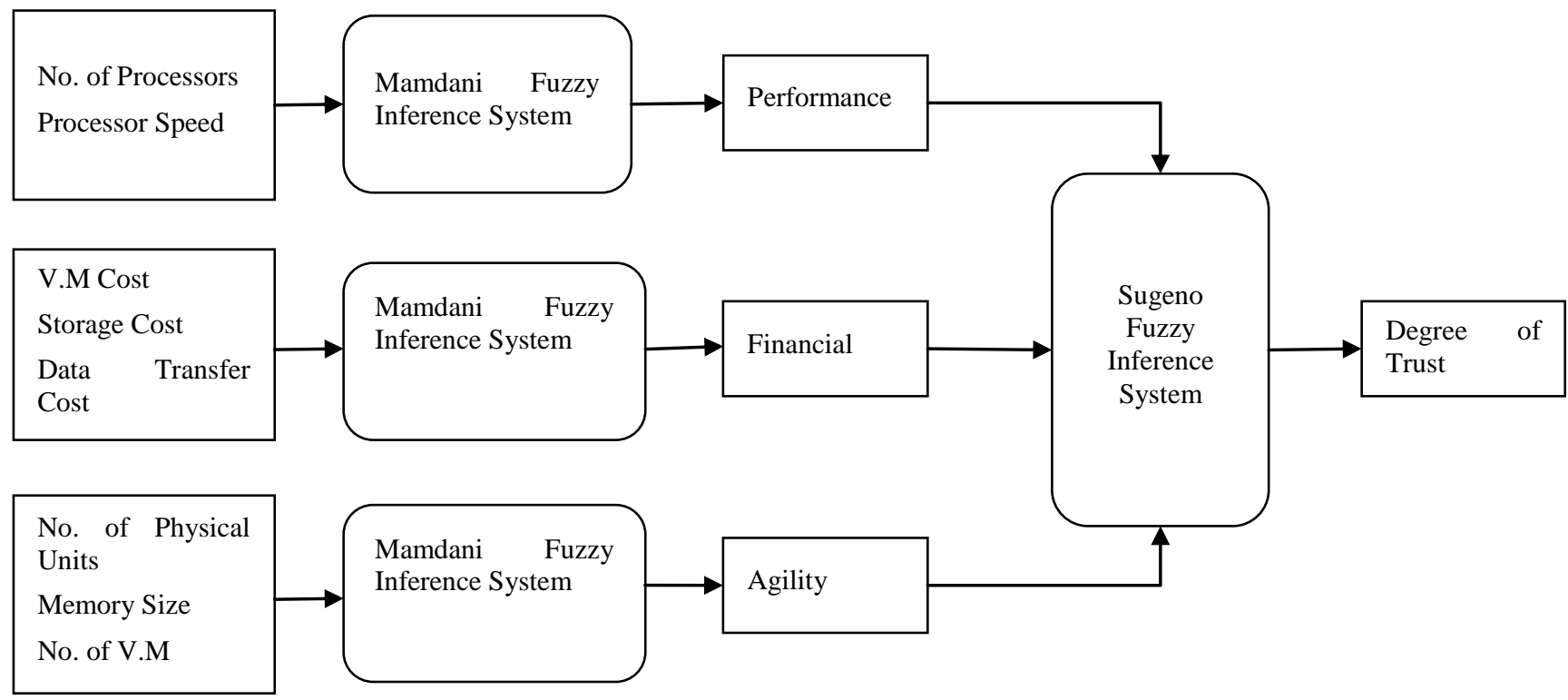

Figure 3: The Block Diagram 
Table 2: Simulation Setup

\begin{tabular}{|c|c|c|c|c|c|c|c|c|c|}
\hline \multirow{2}{*}{$\begin{array}{l}\text { Cloud } \\
\text { Service } \\
\text { Provider }\end{array}$} & \multirow{2}{*}{$\begin{array}{c}\text { No. of } \\
\text { Datacenters }\end{array}$} & \multirow{2}{*}{$\begin{array}{c}\text { No of } \\
\text { Physical } \\
\text { Units }\end{array}$} & \multirow{2}{*}{$\begin{array}{l}\text { Memory } \\
\text { Size } \\
(\mathrm{GB})\end{array}$} & \multirow{2}{*}{$\begin{array}{l}\text { No of } \\
\text { V.Ms }\end{array}$} & \multirow{2}{*}{$\begin{array}{c}\text { No. of } \\
\text { Processors }\end{array}$} & \multirow{2}{*}{$\begin{array}{l}\text { Processor } \\
\text { Speed in } \\
\text { MIPS }\end{array}$} & \multicolumn{3}{|c|}{$\operatorname{Cost}(\$)$} \\
\hline & & & & & & & V.M & Storage & $\begin{array}{c}\text { Data } \\
\text { Transfer }\end{array}$ \\
\hline CSP A & 6 & 6 & 6 & 30 & 24 & 100000 & 0.4 & 0.15 & 0.15 \\
\hline CSP B & 3 & 17 & 1.5 & 15 & 68 & 80000 & 0.1 & 0.25 & 0.09 \\
\hline CSP C & 6 & 6 & 3 & 30 & 24 & 120000 & 0.05 & 0.08 & 0.2 \\
\hline CSP D & 2 & 40 & 4 & 50 & 400 & 160000 & 0.8 & 0.25 & 0.05 \\
\hline CSP E & 3 & 3 & 6 & 150 & 12 & 60000 & 0.5 & 0.15 & 0.09 \\
\hline
\end{tabular}

Simulation Running..

$39 \%$

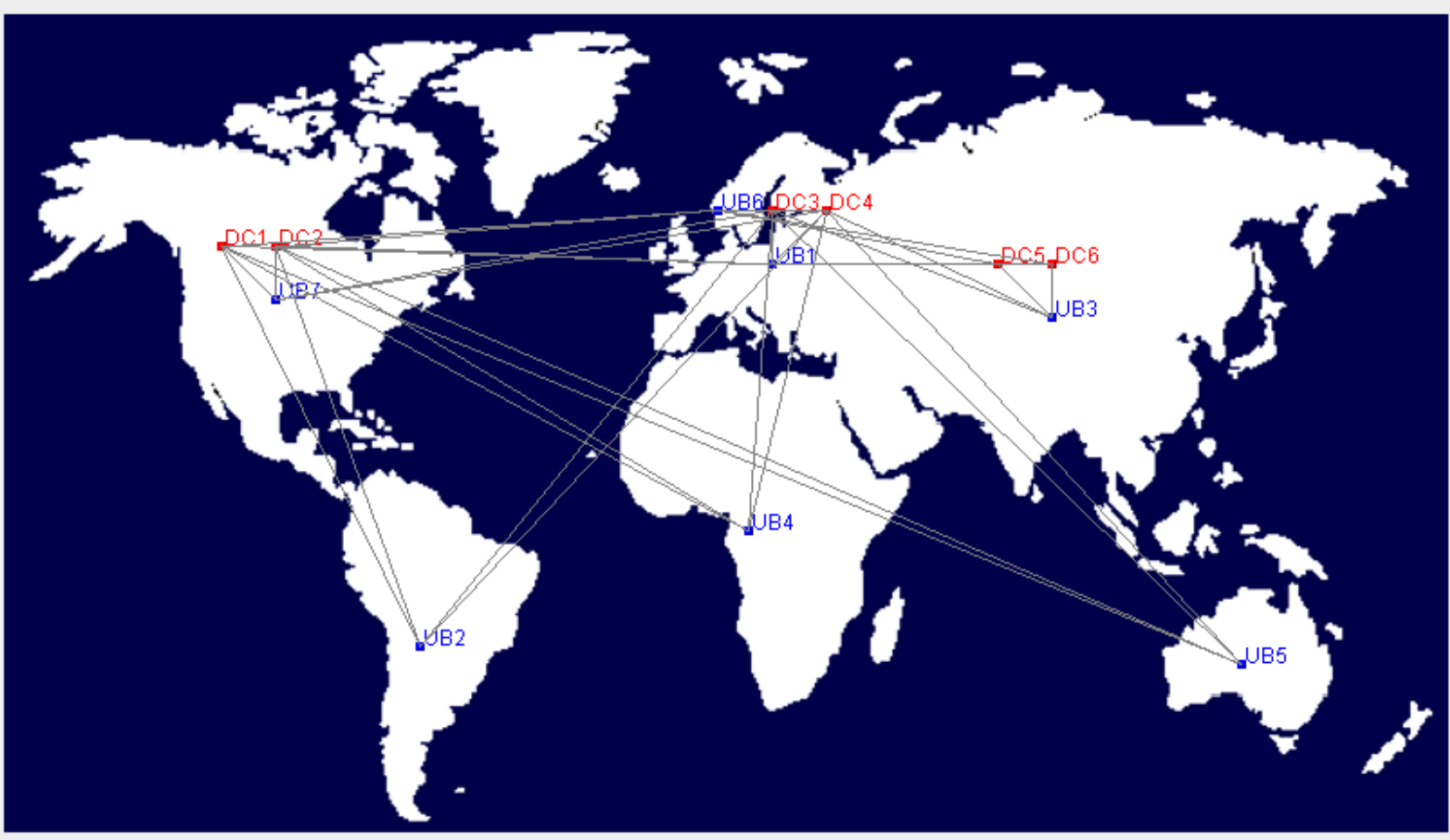

Figure 4: The Cloud Analyst Simulator

\subsection{Cloud Analyst Simulation}

Figure 4 is a sample screen shot showing the simulation in progress. In the figure, UB represents User Bases and DC represents Data Centers. 
Table 3: Results of Cloud Analyst Simulations

\begin{tabular}{|c|c|c|c|c|c|}
\hline \multirow{2}{*}{$\begin{array}{c}\text { Cloud } \\
\text { Service } \\
\text { Provider }\end{array}$} & \multicolumn{2}{|c|}{$\begin{array}{c}\text { Response Time } \\
(\mathrm{ms})\end{array}$} & \multicolumn{2}{c|}{$\begin{array}{c}\text { D.C Processing } \\
\text { Time }(\mathrm{ms})\end{array}$} & $\begin{array}{c}\text { Total } \\
\text { Cost }\end{array}$ \\
\cline { 2 - 5 } & Min & Max & Min & Max & $(\$)$ \\
\hline CSP A & 37.12 & 387.69 & 0.16 & 13.34 & 830.26 \\
\hline CSP B & 37.58 & 385.93 & 0.16 & 13.34 & 361.36 \\
\hline CSP C & 36.89 & 385.61 & 0.14 & 11.26 & 759.01 \\
\hline CSP D & 37.27 & 628.36 & 0.06 & 7.09 & 1140.76 \\
\hline CSP E & 41.46 & 821.02 & 2.08 & 209.18 & 2125.37 \\
\hline
\end{tabular}

Table 3 summarizes the results of the Cloud Analyst for the assumed scenario in Table 2. The Response times and D.C Processing times along with the cost factor in $\$$ listed here show that CSP B is cheaper compared to other CSPs. Section 3.3 describes the estimation of trust value from these results for each CSP using Fuzzy Logic.

\subsection{Fuzzy Logic Implementation}

\subsubsection{Performance}

Performance has two input values namely number of processors and the processor speed as shown in Figure 3. Both have three

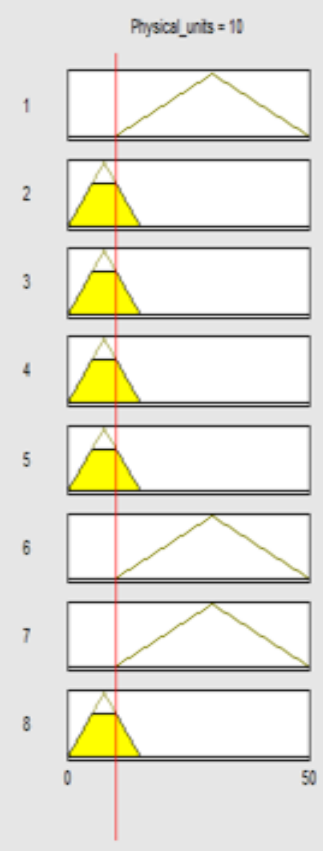

member functions each. The output 'performance' has four member functions low, medium, high, and very high. The outputs obtained from the FIS are CSP A-Low, CSP BMedium, CSP C-Medium, CSP D- Very High, and CSP E-Low.

\subsubsection{Financial}

The financial block also comprises of three inputs namely V.M cost, Storage cost, Data Transfer cost. The 'V.M cost' input has three member functions whereas 'storage cost' and 'Data transfer cost' have two member functions each. The output 'financial' has three member functions low, medium and high. Here a total of nine rules are written. Given the values of CSPs to the financial model we get results as CSP A-High, CSP BMedium, CSP C- High, CSP D-High and CSP E-Medium.

\subsubsection{Agility}

The agility model has three input parameters mapped to one output. Each of the input parameters in Figure 3 has different membership functions. 'Physical units' has two member functions while 'Memory', 'V.M' and output 'Agility' have three member functions each. The range of member functions is chosen based on the actual range of values used. A total of eight rules are written. The output 'Agility' has 3 member functions low, medium and high. When the input related to CSP A is fed to the Matlab's FIS, the Agility comes out as Medium. Similarly for CSP B it is medium, CSP C-low, CSP D-medium, CSP E-medium. Figure 5 shows the sample Rule Viewer when implemented in Matlab.
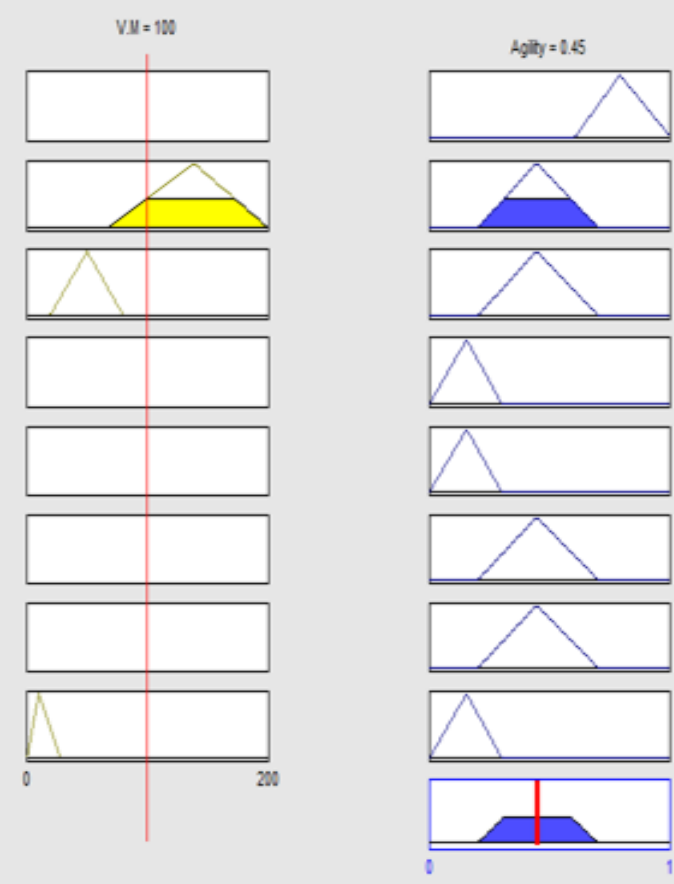

Figure 5: Rule Viewer 


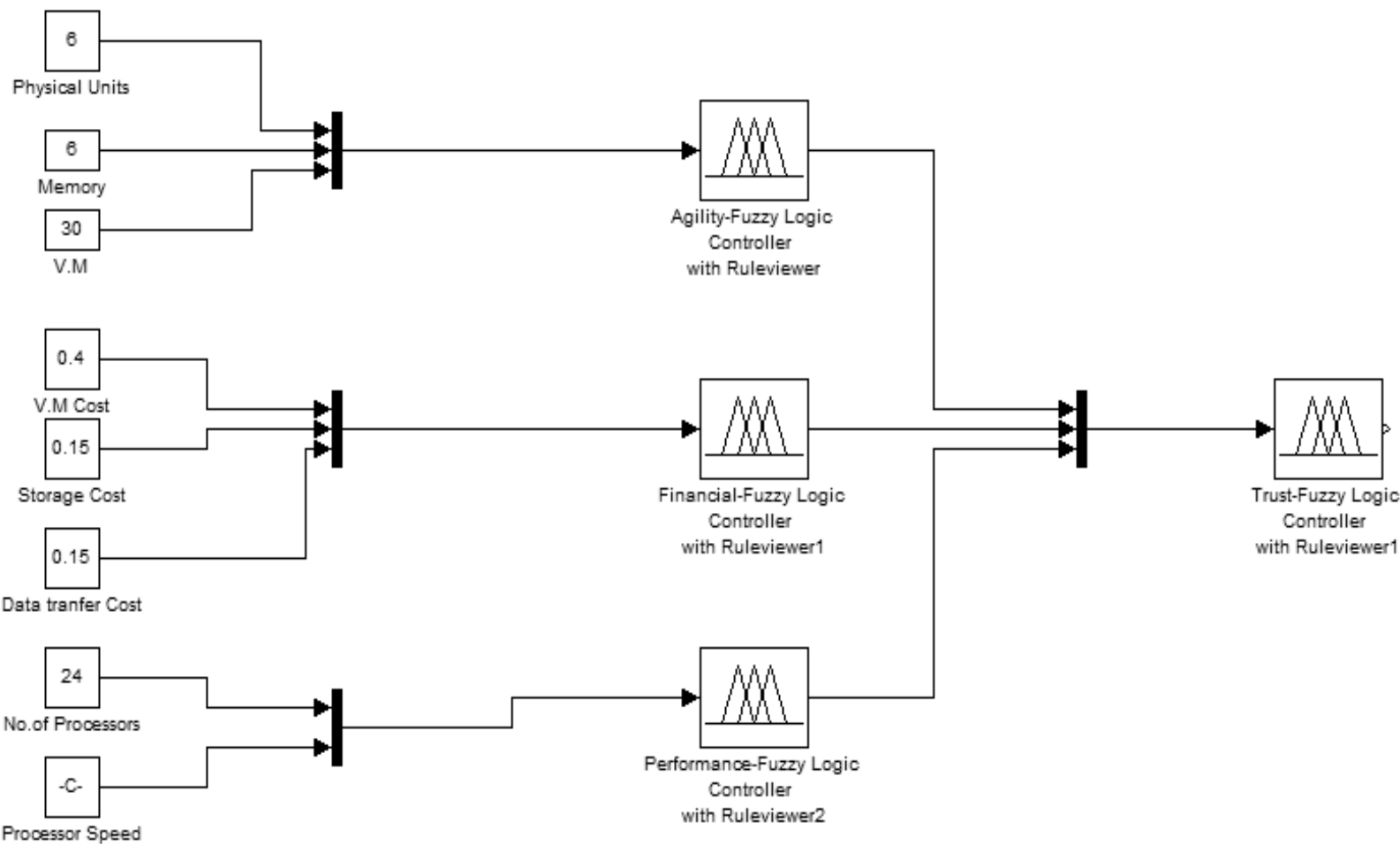

Figure 6: The Simulink Block Diagram

\subsubsection{Trust}

The Trust FIS is the final Fuzzy model which takes the output of the previous three blocks and gives the Trust rating as output. However the fuzzy model chosen here is Sugeno FIS, so the output is a crisp value i.e. one of the five: very poor, poor, good, excellent, and outstanding. This has considerably a large number of rules compared to the previous values due to the increase in number of member functions of input as well as output.

A sample of rules is listed below.

1. If (Agility is low) and (Financial is low) and (Performance is low) then (Trust_Rating is poor) (1)

2. If (Agility is low) and (Financial is medium) and (Performance is low) then (Trust_Rating is very-poor) (1)

3. If (Agility is low) and (Financial is high) and (Performance is low) then (Trust_Rating is very-poor) (1)

4. If (Agility is medium) and (Financial is low) and (Performance is low) then (Trust_Rating is poor) (1)

5. If (Agility is medium) and (Financial is medium) and (Performance is low) then (Trust_Rating is very-poor) (1)

$\begin{array}{ll}\vdots & \vdots \\ \vdots & \vdots\end{array}$

24. If (Agility is medium) and (Financial is high) and (Performance is high) then (Trust_Rating is good) (1)

25. If (Agility is high) and (Financial is low) and (Performance is high) then (Trust_Rating is outstanding) (1)

$\begin{array}{ll}\vdots & \vdots \\ \vdots & \vdots\end{array}$

33. If (Agility is medium) and (Financial is high) and (Performance is very_high) then (Trust_Rating is excellent) (1)

34. If (Agility is high) and (Performance is very_high) then (Trust_Rating is outstanding) (1)

The two fuzzy models: Mamdani and Sugeno are combined together using FIS blocks of Simulink shown in Figure 6, which on execution provide a trust rating using the above rules.

Assuming equal weights for all the rules the trust values estimated for each CSP from above described model are classified in Table 4. But weights can vary as different users would have different expectation from their CSP. Based on this, the rating of the CSP would differ.

Table 4: Trust Value of CSPs

\begin{tabular}{|c|c|c|c|c|}
\hline CSP & Agility & Financial & Performance & Trust \\
\hline CSP A & 0.45 & 0.856 & 0.347 & Poor \\
\hline CSP B & 0.451 & 0.5 & 0.348 & Good \\
\hline CSP C & 0.151 & 0.5 & 0.346 & $\begin{array}{c}\text { Very } \\
\text { Poor }\end{array}$ \\
\hline CSP D & 0.45 & 0.857 & 0.8 & Excellent \\
\hline CSP E & 0.451 & 0.5 & 0.15 & $\begin{array}{c}\text { Very } \\
\text { Poor }\end{array}$ \\
\hline
\end{tabular}

\section{CONCLUSION}

In this paper we have shown that the Inter Domain direct trust value for the CSPs can be estimated using the fuzzy logic tool box which can serve as an indicator for the users to choose a CSP as per their requirement. Also, Cloud Service Providers these days don't offer just a single plan to all consumers. They provide a variety of plans to different types of consumers. So in 
addition to rating different CSPs this model can be used to select a plan based on the users need.

\section{ACKNOWLEDGEMENTS}

We would like to thank Dr. T. S. B. Sudarshan, Chairperson, Department of Computer Science \& Engineering, Amrita School of Engineering, Bengaluru for his guidance and technical inputs.

\section{REFERENCES}

[1] Peter Mell and Timothy Grance. "The NIST Definition of Cloud Computing (Draft) Recommendations of the National Institute of Standards and Technology", NIST Special Publication 800-145, September 2011, 84 pages.

[2] Tripathi, A and Mishra, A. "Cloud Computing Security Considerations", International Conference on Signal Processing, Communications and Computing, India, September 2011, pp. 1-5.

[3] Mohamed Firdhous, Osman Ghazali, and Suhaidi Hassan. "Trust and Trust Management in Cloud Computing - A Survey", Internetworks Research Group, Universiti Utara Malaysia, Technical Report, February 2011.

[4] Xiaodong Sun, Guiran Chang and Fengyun Li. "A Trust Management Model to Enhance Security of Cloud Computing Environments", Second International Conference on Networking and Distributed Computing, China, September 2011, pp. 244-248.
[5] Mohammed Alhamad, Tharam Dillon and Elizabeth Chang. "A Trust-Evaluation Metric for Cloud Applications", International Journal of Machine Learning and Computing, Volume 1, Number 4, October 2011, pp. 416-421.

[6] Cloud Service Measurement Index Consortium (CSMIC) "Service Measurement Index Version 1.0" (PDF), USA, September 2011.

[7] Garg, S.K., Versteeg, S and Buyya, R. "SMICloud: A Framework for Comparing and Ranking Cloud Services", Fourth International Conference on Utility and Cloud Computing, Australia, December 2011, pp. 210-218.

[8] Cloud Security Alliance, "Top Threats to Cloud Computing V1.0", March 2010.

[9] Jensen M, Schwenk J, Gruschka N and Iacono, L.L. "On Technical Security Issues in Cloud Computing", International Conference on Cloud Computing, Germany, September 2009, pp. 109-116

[10] Wickremasinghe, B, Calheiros R.N and Buyya, R. "CloudAnalyst: A CloudSim-Based Visual Modeller for Analyzing Cloud Computing Environments and Applications" 24th International Conference on Advanced Information Networking and Applications, Australia, April 2010, pp. 446-452.

[11] http://www.mathworks.com/help/pdf_doc/fuzzy/fuzzy.pdf" Fuzzy Logic Toolbox ${ }^{\mathrm{TM}}$ User's Guide. 